\title{
Simulation design of fuze warhead system of air defense missile at very low altitude
}

\author{
Qi Zhao*, Guangyu Du, Bin Zhang, Yanbin Zhai, and Yuruo Shi \\ Beijing Institute of Electronic System Engineering, Beijing 100854, China
}

\begin{abstract}
A novel simulation method for fuze warhead system (FWS) at very low altitude flight is proposed to solve adaptability issues of the traditional one in the naval battle. Firstly, a simulation system framework is presented. Then the detailed implementation of a novel general fuze model, a novel sea echo model and a novel warhead dynamic effectiveness power field algorithm including the simulation system are presented. Finally, simulation results show good performance of the proposed method. The proposed method can simulate the echo signal when the complex fuze antennas detect target and the sea at the same time, and can truly reflect the target positions hit by the warhead fragments. The proposed method can solve the existing problems in the FWS simulation system.
\end{abstract}

Keywords: fuze warhead system (FWS), simulation, fuze warhead matching, sea echo, fragment trajectory drifting.

DOI: $10.21629 / J S E E .2017 .03 .17$

\section{Introduction}

The very low altitude penetration is a common means of attack in modern naval battle, therefore the air defense missile's fuze warhead system (FWS) needs not only to prevent early explosion caused by the sea echo, but also to detect the target signal effectively and detonate the warhead timely to hit the target [1].

Pulse Doppler radio fuze is commonly used in air defense missile to transmit radio wave and receive scattering signal to detect the target. Without any special method, it will cause early explosion when the missile flying at the very low altitude receives sea clutter and the target signal at the same time.

Currently the methods used to design and verify the FWS especially at the very low altitude contain: target test, fuze slide rail test, fuze hung on the helicopter flight test and full digital simulation test of FWS. The specific characteristics of these tests are shown as follows:

(i) The size of target test samples is small and the

Manuscript received January 06, 2016

*Corresponding author. parameters that can be measured are not enough. (ii) Fuze slide rail test uses the high-speed rocket engine to push the fuze to slide on the rail and metal mesh to simulate sea echo. On one hand, due to the scattering properties and the size limit of metal mesh, there is a large gap between the true sea echo and the simulated echo. On the other hand, due to the limit of test field area, the type of sea situation, flight attitude and velocity of fuze can only be set to some very limited states. (iii) Fuze hung on the helicopter flight test cannot test the capabilities of fuze extracts target signal from mixed ones because there is none in the test. In addition, it is difficult to simulate the real state of the missile flying at very low altitude because of the limitation of helicopter's speed and altitude.

In view of the limitations of the above three kinds of tests, the fully digital simulation test of FWS has become important to overcome the limitation of traditional methods because it has advantages of low cost, very short test period, evaluation and analysis in various sea and encounter conditions.

However, currently fully digital simulation platforms for FWS at very low altitude are not perfect. Some fuze design means were summarized in [1]. A method of sea echo signal calculation in a particular antenna pattern condition was described in [2]. The multi-scattering centers modeling and simulation method of the near field target was described in $[3,4]$, but the modeling method of the sea clutter was not mentioned. Reference [5] had done a great job on the design of the FWS simulation system, but the ground and sea clutter model can be further studied in the very low altitude simulation. References [6,7] did a lot of meaningful work on the phenomenon that the target's positions were hit by warhead's fragments drift because of the air resistance, however, the precision of the algorithm can be promoted.

This paper is organized as follows. A novel modeling and simulation system of FWS composed by the pulse Doppler fuze and fragment warhead especially for the very low altitude is presented in this paper. The FWS simulation 
system is introduced in Section 2. Details of a novel near field scattering characteristics of sea clutter modeling algorithm are also proposed in this Section. Based on this and the targets' multi-scattering centers modeling algorithm, the sea clutter and target mixed echo are proposed. Combination of a novel general antenna pattern model described in this section, mixed echo of various targets detected by different fuzes can be calculated. A novel algorithm of warhead's fragments' trajectories drifting because of the air resistance suitable for the large-scale calculation is also proposed in this section. Then in Section 3, a particular FWS math model designed for very low altitude is embedded in the platform to evaluate its performance after the simulation verification of the sea clutter model and fragments' trajectories drifting model, then the design is improved aiming at the existing problem and verified again. Conclusions are reported in Section 4.

\section{Fully digital FWS simulation system}

\subsection{Design overview}

The simulation system of FWS is divided into ten modules, layered and modularized from the division of functions and data management point of view in accordance with the modular object-oriented design idea (see Fig. 1).

(i) System control module

System control module is responsible for coordinating the operation of the entire system including processing the system operation parameters, mobilizing the various modules timely and transferring the data between them.

(ii) Missile/target motion module

Depending on the target's and the missile's trajectories, velocities and attitudes, this module calculates not only the transformation matrix such as missile to the relative velocity coordinate system, target to the relative velocity coordinate system, launching to the relative velocity coordinate system, but also the attitudes, position, velocity in the missile and target relative velocity coordinate system in each period.

(iii) Target near field multi-scattering centers' module

The concept of multi-scattering centers [8-10] provides a theoretical basis and method for modeling of the radar near field target, and it simplifies the extremely complex computational and analytical efforts of the electric magnetic scattering. The typical target multi-scattering centers' data for this module are obtained through the combination of theoretical calculation and actual measurement.

(iv) Sea echo module

This module presents a sea clutter echo calculation method suitable for pulse Doppler fuze. The backscattering coefficient of the sea from the different grazing angle and sea condition used for this module is obtained according to the theoretical calculation and the experimental data correction. The sea surface is divided according to the missile flying condition, antenna radiation ability and simulation accuracy setting, and then the module vector synthesizes every piece of sea surface signal to calculate the entire ones. The echo mixed by the target and sea is presented base on the multi-scattering centers and this algorithm.

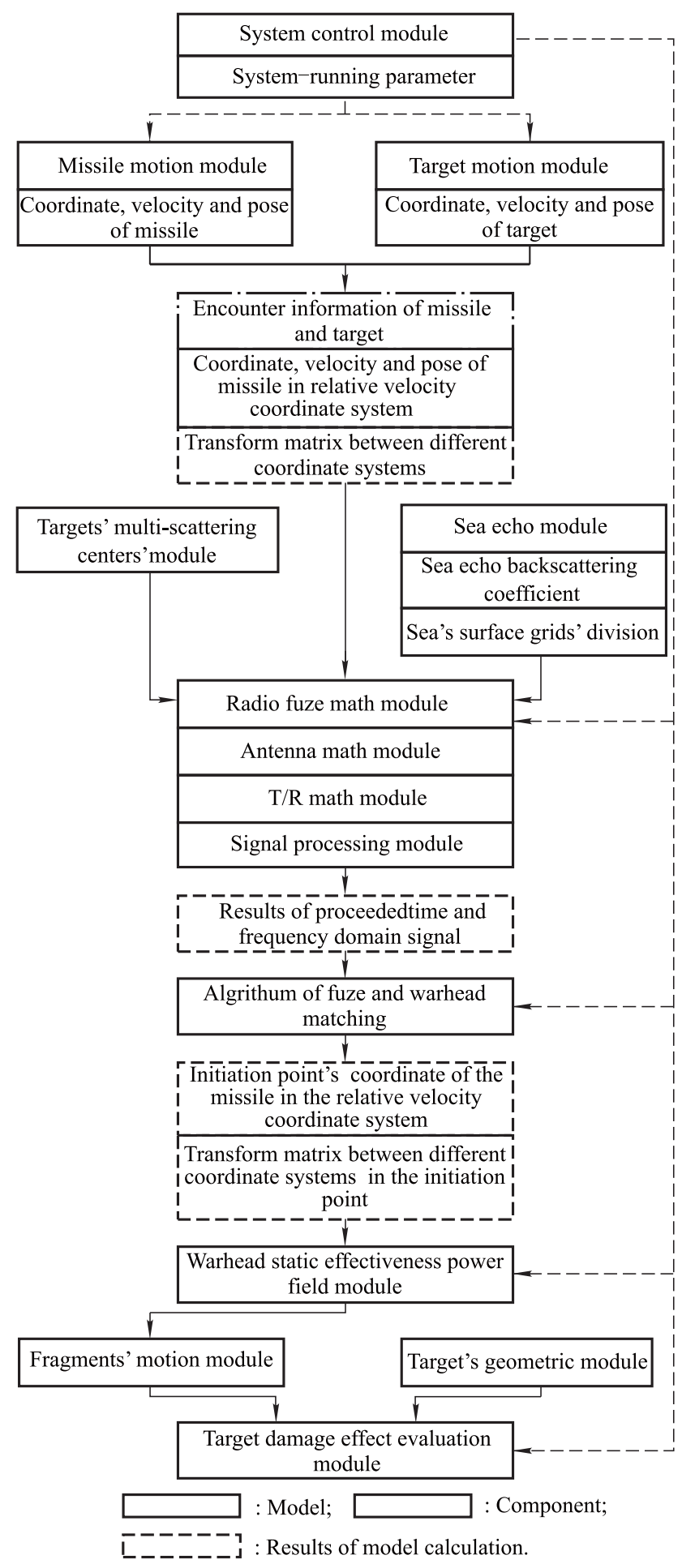

Fig. 1 Block diagram of FWS simulation system 
(v) Radio fuze mathematical module

Radio fuze mathematical module is part of the simulation platform to be detected. The simulation system can be embedded in a different fuze mathematical model to detect whether the fuze signal processing algorithm and hardware performance meet the requirements. The improvements of the software or hardware can be designed for local problems and the effect of improvement can be verified by simulation. The fuze mathematical module contains antenna module, transmitter and receiver modules and signal processing module. This paper presents a novel general fuze antenna mathematical model to calculate echoes according to different antenna patterns.

(vi) Warhead static explosion power field module

The warhead static explosion power field module is the basis to calculate the target destructive effect. This module includes the each fragment's initial position, shape, material, weight and velocity vector, and the equivalent amount of TNT charge weight. This paper does not focus on this module.

(vii) Fuze warhead matching algorithm module

The fuze warhead matching algorithm module which ensures the coordination of the fuze detection area and the warhead dynamic power field in order to achieve the target maximum destructive effect is the core part of FWS. This module can be embedded and replaced in the simulation system to achieve the effect and analyze the results to improve the algorithm. This paper focuses on the simulation design of FWS, so this module is not the emphasis.

(viii) Fragments' trajectory module

This module calculates each fragment's trajectory based on the warhead static explosion power field, missile initiation position, velocity, altitude and air resistance at different altitudes. A novel algorithm precise and suitable for the large-scale calculation is proposed.

(ix) Target's geometric/target's damage effect evaluation module

Target's geometric module builds three-dimensional geometric models based on the real targets. Target damage effect evaluation module calculates the number of fragments hitting target based on the target geometric and the fragments' trajectories.

\subsection{Fuze echo mathematical model}

The fuze echo mathematical model including the fuze model, target echo model and sea clutter echo model is the core of FWS. A novel general model which can simulate a variety of different patterns, targets and sea conditions in the complex encounter situation is proposed in this section.

\subsubsection{Fuze mathematical model}

Antenna pattern determines the fuze detection area, and the fuze antenna often consists of multiple sets of antennas, so the detection area is very complicated. This section presents a simulation method to restore the threedimensional pattern of the antenna using two-dimensional pattern which has strong versatility and can reflect the main and side lobes' detection area.

The modeling method used in this section is shown in Fig. 2.

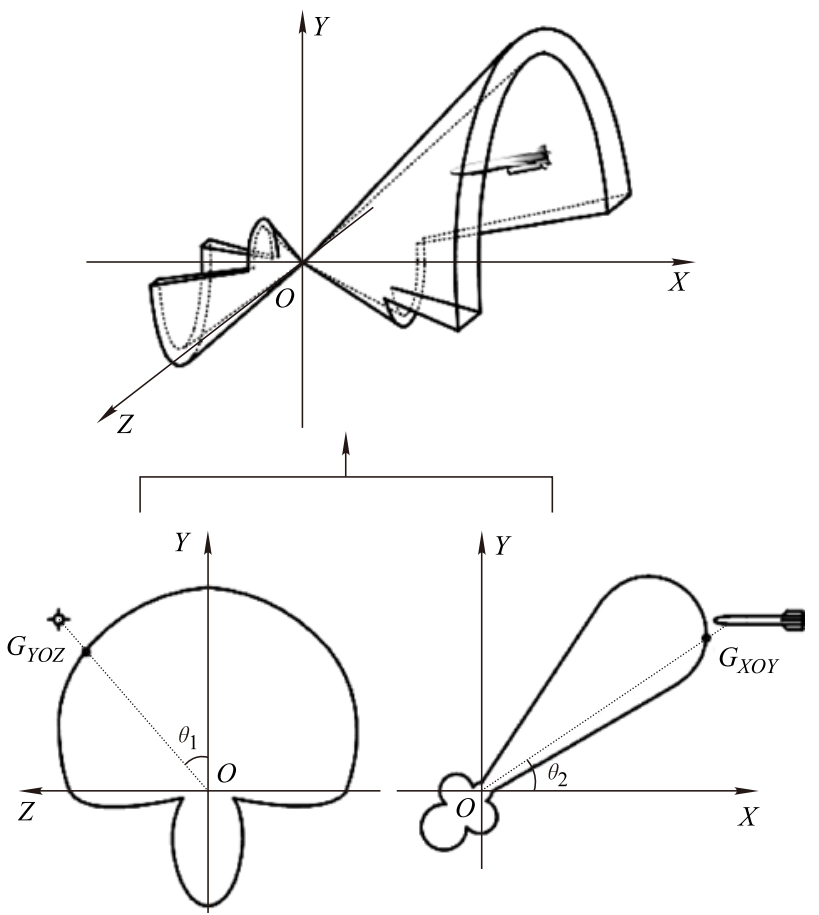

Fig. 2 Antenna pattern 3D model

Target coordinates $\boldsymbol{P}_{T A}$ in the antenna coordinate system can be given as

$$
\boldsymbol{P}_{T A}=\left[\begin{array}{c}
x_{T A} \\
y_{T A} \\
z_{T A}
\end{array}\right]=\boldsymbol{E}_{T 2 A}\left[\begin{array}{c}
x_{T} \\
y_{T} \\
z_{T}
\end{array}\right]
$$

where $\left[x_{T}, y_{T}, z_{T}\right]^{\mathrm{T}}$ is the single scattering center coordinates of the target, $\boldsymbol{E}_{T 2 A}$ is the target to the missile body coordinate system transformation matrix.

The overall gain of the single antenna can be given by

$$
G\left(\theta_{1}, \theta_{2}\right)=G_{Y O Z}\left(\theta_{1}\right) * G_{X O Y}\left(\theta_{2}\right)
$$

where $\theta_{1}$ is the angle that the target single scattering centers in the $Y O Z$ plane of the antenna coordinate system, $\theta_{2}$ is the angle between the target single scattering center and the antenna axis, $\theta_{1}$ and $\theta_{2}$ can be given by

$$
\theta_{1}=\arctan \left(\frac{z_{T A}}{y_{T A}}\right)
$$




$$
\theta_{2}=\left\{\begin{array}{l}
2 \pi-\arccos \left(\frac{x_{T A}}{\left|P_{T A}\right|}\right), \quad \frac{\pi}{2}<\theta_{1}<\frac{3 \pi}{2} \\
\arccos \left(\frac{x_{T A}}{\left|P_{T A}\right|}\right), \text { otherwise }
\end{array} .\right.
$$

\subsubsection{Target multi-scattering centers echo model}

Target echo signal simulation is the basis and core of FWS. An effective way of target echo simulation called multiscattering centers or highlight was described in $[11,12]$. This method can be explained as air targets composed by a fixed number of scattering centers, that is, the number of scattering centers and the position of each individual air targets are fixed. The scattering intensity depends on illumination and receiving sight.

The target echo signal at each sampling time can be given as

$$
\begin{gathered}
S_{r}(t)=\sum_{k=-\infty}^{+\infty} \sum_{i=0}^{I-1} \operatorname{Rect}\left[\frac{t-\tau_{j}-i T_{r}-k I T_{r}}{T_{p}}\right] \boldsymbol{C}_{i} . \\
\sum_{j=1}^{J} G\left(\theta_{1 j}, \theta_{2 j}\right) S_{j} \frac{\sqrt{\sigma_{T j}}}{R_{j}^{2}} \cos \left(\frac{4 \pi R_{j}}{\lambda}+\varphi_{0}\right)
\end{gathered}
$$

where $\sigma_{j}$ is the $j$ th scattering center's radar cross section (RCS) which can be obtained by using the target multiscattering center database, $G\left(\theta_{j}, \delta\right)$ is the antenna pattern, $R_{j}$ is the distance between the target's $j$ th scattering center and the fuze at time $t, \lambda$ is the wavelength of the fuze, $S_{j}$ is the sensitivity according to the $R_{T_{j}}, C_{i} \in\{1,-1\}$ is a pseudo-random binary phase-coded phase modulation factor, $\tau_{i}$ is the round-trip travel time of electromagnetic waves between the target's $j$ th scattering center and the fuze, $I$ is the pseudo-random code length, $T_{r}$ is the fuze transmitter pulse repetition period, $T_{p}$ is the transmitter pulse width, $\varphi_{0}$ is the initial phase of the carrier wave,

$$
\operatorname{Rect}_{i}\left(\frac{t}{T}\right)=\left\{\begin{array}{ll}
1, & -T_{p} / 2<t<T_{p} / 2 \\
0, & \text { otherwise }
\end{array} .\right.
$$

\subsubsection{Sea clutter echo model}

\section{(i) Calculation guideline}

The method proposed in [2] has limitation that it can only calculate the sea echo signal under certain situation. This method is only suitable for the lateral narrow beam antenna considering the influence of main lobe and side lobe in ideal conditions, it cannot simulate different types of antennas. The FWS simulation system needs an algorithm which not only should work with the antenna pattern changing or the non-ideal main lobe and side lobe being used, but also can simulate the mixed signal of the target and the sea echo simultaneously.
In order to better demonstrate the conditions that the fuze antenna illuminates the sea, this section presents a novel algorithm which can calculate the sea and the target echo together. The basic idea of the algorithm of sea echo calculating is divided meticulously into small meshes called surface element according to the fuze irradiation area of sea, and then each surface element of sea surface scattering intensity is synthesized to form the whole sea echo.

The calculation of the sea echo is performed in three steps.

Step 1 Use the missile real-time flight height to determine the circular area of the sea surface required to compute.

Step 2 Divide the sea surface required to compute into meshes according to the preset angle interval $\Delta \theta$ and radius step $\Delta R$.

Step 3 Read the corresponding sea echo RCS value from the database depending on sea conditions, wind direction, and the grazing angle $\alpha_{n}$.

(ii) Sea surface mesh division algorithm

The division algorithm of sea surface mesh is shown in Fig. 3. The circular area needed to be calculated in different flight altitudes is not the same, and it is becoming bigger as the flight altitude is getting lower.

The circular area mentioned above can be given as

$$
S_{C}=\sum_{n=1}^{N} \sum_{m=1}^{M} S_{C_{m, n}}
$$

where $S_{C_{m, n}}$ is the area of each element which can be given as

$S_{C_{m, n}}= \begin{cases}\frac{\pi \Delta R^{2}(2 n-1) \Delta \theta}{360}, \quad m<M \\ \frac{\pi \Delta R^{2}(2 n-1)(360-\Delta \theta(m-1))}{360}, \quad m=M\end{cases}$

where $\Delta R$ is the preset radius step, $\Delta \theta$ is the preset angle step, $M$ is the size of angle division, $N$ is the size of the radius division, and they can be given by

$$
\left\{\begin{array}{l}
M=\operatorname{ceil}\left(\frac{360^{\circ}}{\Delta \theta}\right) \\
N=\operatorname{ceil}\left(\frac{\sqrt{R_{D 3}^{2}-H_{i}^{2}}}{\Delta R}\right)
\end{array}\right.
$$

where $R_{D 3}$ is the fuze cut-off detection range, $H_{i}$ is the flight altitude.

(iii) Sea echo calculation

According to the sea surface backscattering coefficient 
database, (2) and (6), we have

$$
\begin{aligned}
& S_{r}(t)= \sum_{k=-\infty}^{+\infty} \sum_{i=0}^{I-1} \operatorname{Rect}\left[\frac{t-\tau_{j}-i T_{r}-k I T_{r}}{T_{p}}\right] \boldsymbol{C}_{i} . \\
& \sum_{n=1}^{N} \sum_{m=1}^{M} G\left(\theta_{1 m, n}, \theta_{2 m, n}\right) S_{m, n} \cdot \\
& \frac{\sqrt{\sigma_{S_{m, n}} S_{C_{m, n}}}}{R_{S_{m, n}}^{2}} \cos \left(\frac{4 \pi R_{S_{m, n}}}{\lambda}+\varphi_{m, n}\right)
\end{aligned}
$$

where $\varphi_{m, n}$ is the phase of each surface element, which can be given as

$$
\varphi_{m, n}=4 \pi \sqrt{H_{i}^{2}+(m-0.5)^{2} \Delta R^{2}} / \lambda
$$

where $R_{S_{m, n}}$ is the range between fuze antenna and each sea surface element's center which can be given as

$$
R_{S_{m, n}}=\sqrt{H_{i}^{2}+(m-0.5)^{2} \Delta R^{2}}
$$

where $\sigma_{S_{m, n}}$ is related to the wind direction, the sea condition preseted at the beginning of the simulation, and the grazing angle $\alpha_{m, n}$ which can be given as

$$
\alpha_{m, n}=\arctan \left(\frac{H_{i}}{(m-0.5) \Delta R}\right) .
$$

(iv) Target and sea echo model

According to (5) and (9), we have

$$
\begin{gathered}
S_{r}(t)=\sum_{k=-\infty}^{+\infty} \sum_{i=0}^{I-1} \operatorname{Rect}\left[\frac{t-\tau_{j}-i T_{r}-k I T_{r}}{T_{p}}\right] \boldsymbol{C}_{i} . \\
{\left[\sum_{j=1}^{J} G\left(\theta_{1 j}, \theta_{2 j}\right) S_{j} \frac{\sqrt{\sigma_{T j}}}{R_{j}^{2}} \cos \left(\frac{4 \pi R_{j}}{\lambda}+\varphi_{0}\right)+\right.} \\
\sum_{n=1}^{N} \sum_{m=1}^{M} G\left(\theta_{1 m, n}, \theta_{2 m, n}\right) S_{m, n} \frac{\sqrt{\sigma_{S_{m, n}} S_{C_{m, n}}}}{R_{S_{m, n}}^{2}} \\
\left.\left(\frac{4 \pi R_{S_{m, n}}}{\lambda}+\varphi_{0}\right)\right] .
\end{gathered}
$$

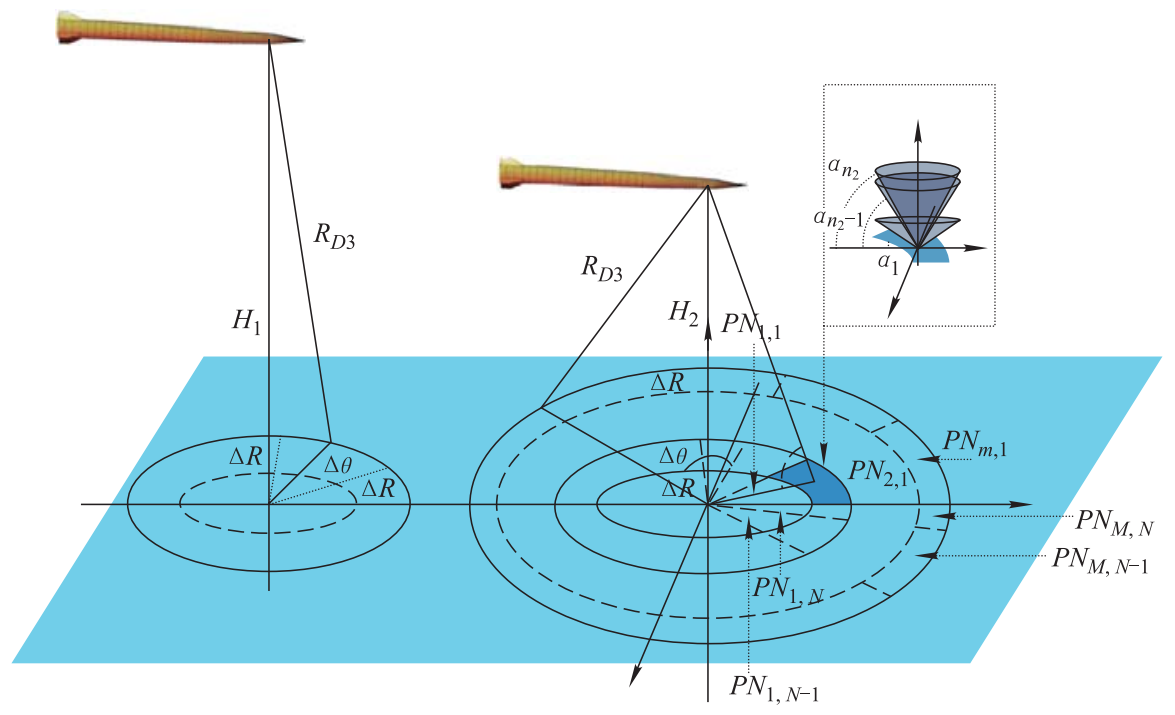

Fig. 3 Division mesh of the sea surface

\subsection{Warhead dynamic effectiveness power field model}

The calculation of the fragments hitting the target can no longer be considered as the uniform linear motion in the relative velocity coordinate system because the air resistance cannot be ignored at very low altitude. This process can be considered that the target velocity is constant, but the fragments' decrease, and fragments' flying trajectories in coordinated relative velocity are no longer straight lines but curves.

The average speed method mentioned in [4] cannot accurately calculate the change of position where the frag- ment hits the target. The method used in [5] was not precise enough because it assumed that the missile did not roll and the target was in flat flight. The variation law of the fragment trajectory mentioned in [7] was limited to the fragments whose initial velocity was perpendicular to the axial direction of the missile and did not calculate the positions hit by the fragments.

FWS is often faced with hundreds of thousands of largescale computation, so we need to weigh on the simulation time and accuracy. Then this algorithm needes to freely set the simulation precision to meet the performance requirements. Aiming at the requirement, a novel algorithm 
is proposed to calculate the target's positions hit by warhead's fragments drifting.

Fragment's initial velocity in the ground coordinate system is synthesized by the fragment's muzzle velocity in the missile coordinate system and the missile's after warhead detonation. Fragment velocity's direction in the ground coordinate system does not change while the value decreases according to the fragment flying distance because of the air resistance. The target's movement can be seen as a process of uniform linear motion in fragments flying time. The fragment and the target are both in movement in the ground coordinate system, thus solving the target's point hit by the fragment is suitable in the relative velocity coordinate system. We only need to calculate the fragment's velocity and trajectory because the target is static in this coordinate system. The calculation process is shown in Fig. 4, and the detailed calculation steps are as follows.

Step 1 Obtain the input parameters at the warhead detonation time as follows:

(i) Missile velocity in launching coordinate system can be given as

$$
V_{M-L a u n C r d}=
$$

$\left[V X_{M_{-} L a u n C r d}, V Y_{M_{-} L a u n C r d}, V Z_{M_{-} L a u n C r d}\right]$.

(ii) Target velocity in the launching coordinate system can be given as

$$
\boldsymbol{V}_{T_{-} \text {LaunCrd }}=
$$

$\left[V X_{T_{-} L a u n C r d}, V Y_{T_{-} L a u n C r d}, V Z_{T \_L a u n C r d}\right]$.

(iii) Fragments flying interval time $\Delta t_{a t n}$.

(iv) The missile altitude at the warhead detonation time $H_{M}$.

(v) Transformation matrix from the relative velocity to the launching coordinate system $\boldsymbol{E}_{V 2 L}$.

(vi) Transformation matrix from the relative velocity to the missile body coordinate system $\boldsymbol{E}_{V 2 M}$.

(vii) Transformation matrix from the target body to the relative velocity coordinate system $\boldsymbol{E}_{T 2 V}$.

Step 2 Obtain the fragment's initial coordinate $\boldsymbol{P}_{\text {Init }}\left(n_{0}\right)$ and velocity $\boldsymbol{V}_{\text {Init }}\left(n_{0}\right)$.

The fragment's initial coordinate in the velocity coordinate system can be given by

$$
\begin{gathered}
\boldsymbol{P F}_{\text {Init_VrelCrd }}\left(n_{0}\right)=\left[\begin{array}{l}
P F X_{\text {Init_VrelCrd }}\left(n_{0}\right) \\
P F Y_{\text {Init_VrelCrd }}\left(n_{0}\right) \\
P F Z_{\text {Init_VrelCrd }}\left(n_{0}\right)
\end{array}\right]= \\
\boldsymbol{E}_{V 2 M}^{-1} \boldsymbol{P} \boldsymbol{F}_{\text {Init_MslCrd }}\left(n_{0},:\right)+\boldsymbol{P M}_{\text {Init_VrelCrd }}= \\
\boldsymbol{E}_{V 2 M}^{-1}\left[\begin{array}{l}
P F X_{\text {Init_MslCrd }}\left(n_{0}\right) \\
P F Y_{\text {Init_MslCrd }}\left(n_{0}\right) \\
P F Z_{\text {Init_MslCrd }}\left(n_{0}\right)
\end{array}\right]+
\end{gathered}
$$

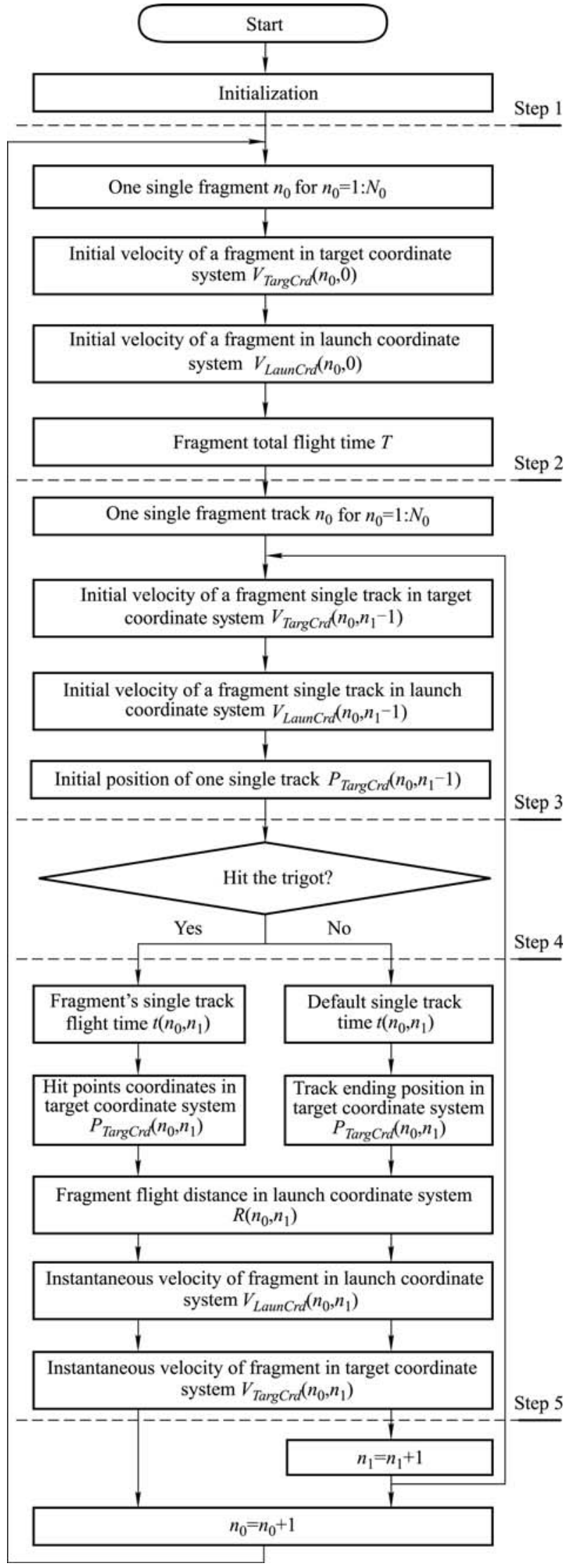

Fig. 4 Steps of fragment trajectory calculation 


$$
\left[\begin{array}{l}
P M X_{\text {Init_VrelCrd }} \\
P M Y_{\text {Init_VrelCrd }} \\
P M Z_{\text {Init_VrelCrd }}
\end{array}\right]
$$

where $\boldsymbol{P} \boldsymbol{F}_{\text {Init_MslCrd }}\left(n_{0}\right)$ is the fragment's initial coordinate in the missile body system, $\boldsymbol{P} \boldsymbol{M}_{\text {Init_VrelCrd }}$ is the missile's detonation position in the relative velocity coordinate system.

According to (16), the fragment's initial coordinate in the target body system can be given by

$$
\begin{gathered}
\boldsymbol{P F}_{\text {Init_TargCrd }}\left(n_{0}\right)=\left[\begin{array}{l}
P F X_{\text {Init_TargCrd }}\left(n_{0}\right) \\
P F Y_{\text {Init_TargCrd }}\left(n_{0}\right) \\
P F Z_{\text {Init_TargCrd }}\left(n_{0}\right)
\end{array}\right]= \\
\boldsymbol{E}_{T 2 V}^{-1}\left[\begin{array}{l}
P F X_{\text {Init_VrelCrd }}\left(n_{0}\right) \\
P F Y_{\text {Init_VrelCrd }}\left(n_{0}\right) \\
P F Z_{\text {Init_VrelCrd }}\left(n_{0}\right)
\end{array}\right] .
\end{gathered}
$$

The relative velocity $\boldsymbol{V}_{\text {rel }}$ can be given by

$$
\begin{gathered}
\boldsymbol{V}_{\text {rel }}=\left[\begin{array}{c}
V X_{r e l} \\
V Y_{r e l} \\
V Z_{r e l}
\end{array}\right]= \\
\left|\left[\begin{array}{c}
V X_{M_{-} L a u n C r d} \\
V Y_{M_{-} \text {LaunCrd }} \\
V Z_{M_{\text {_LaunCrd }}}
\end{array}\right]-\left[\begin{array}{c}
V X_{T \_L a u n C r d} \\
V Y_{T \_L a u n C r d} \\
V Z_{T \_L a u n C r d}
\end{array}\right]\right| .
\end{gathered}
$$

The fragment initial velocity in the relative velocity coordinate system $\boldsymbol{V} \boldsymbol{F}_{\text {Init_V relCrd }}\left(n_{0}\right)$ can be given by

$$
\begin{gathered}
\boldsymbol{V} \boldsymbol{F}_{\text {Init_VrelCrd }}\left(n_{0}\right)=\left[\begin{array}{l}
V F X_{\text {Init_VrelCrd }}\left(n_{0}\right) \\
V F Y_{\text {Init_VrelCrd }}\left(n_{0}\right) \\
V F Z_{\text {Init_VrelCrd }}\left(n_{0}\right)
\end{array}\right]= \\
\boldsymbol{E}_{M 2 V}\left[\begin{array}{c}
V F X_{\text {Init_MslCrd }}\left(n_{0}\right) \\
V F Y_{\text {Init_MslCrd }}\left(n_{0}\right) \\
V F Z_{\text {Init_MslCrd }}\left(n_{0}\right)
\end{array}\right]+\left[\begin{array}{c}
V X_{\text {rel }} \\
V Y_{\text {rel }} \\
V Z_{\text {rel }}
\end{array}\right] \cdot
\end{gathered}
$$

The fragment initial velocity in the target coordinate system $\boldsymbol{V} \boldsymbol{F}_{\text {Init_TargCrd }}\left(n_{0}\right)$ can be given by

$$
\begin{gathered}
\boldsymbol{V} \boldsymbol{F}_{\text {Init_TargCrd }}\left(n_{0}\right)=\left[\begin{array}{l}
V F X_{\text {Init_TargCrd }}\left(n_{0}\right) \\
V F Y_{\text {Init_TargCrd }}\left(n_{0}\right) \\
V F Z_{\text {Init_TargCrd }}\left(n_{0}\right)
\end{array}\right]= \\
\boldsymbol{E}_{T 2 V}^{-1}\left[\begin{array}{l}
V F X_{\text {Init_VrelCrd }}\left(n_{0}\right) \\
V F Y_{\text {Init_VrelCrd }}\left(n_{0}\right) \\
V F Z_{\text {Init_VrelCrd }}\left(n_{0}\right)
\end{array}\right]
\end{gathered}
$$

where $\boldsymbol{V} \boldsymbol{F}_{\text {Init_MslCrd }}\left(n_{0}\right)$ is the fragment velocity in the missile body coordinate system.

The fragment initial velocity in the launching coordinate system $\boldsymbol{V} \boldsymbol{F}_{\text {Init_LaunCrd }}\left(n_{0}\right)$ can be given by

$$
\boldsymbol{V} \boldsymbol{F}_{\text {Init_LaunCrd }}\left(n_{0}\right)=\left[\begin{array}{l}
V F X_{\text {Init_LaunCrd }}\left(n_{0}\right) \\
V F Y_{\text {Init_LaunCrd }}\left(n_{0}\right) \\
V F Z_{\text {Init_LaunCrd }}\left(n_{0}\right)
\end{array}\right]=
$$

$$
\begin{gathered}
\boldsymbol{E}_{V 2 L} \boldsymbol{E}_{V 2 M}^{-1}\left[\begin{array}{l}
V F X_{\text {Init_MslCrd }}\left(n_{0}\right) \\
V F Y_{\text {Init_MslCrd }}\left(n_{0}\right) \\
V F Z_{\text {Init_MslCrd }}\left(n_{0}\right)
\end{array}\right]+ \\
{\left[\begin{array}{c}
V X_{M \_L a u n C r d} \\
V Y_{M \_L a u n C r d} \\
V Z_{M \_L a u n C r d}
\end{array}\right] .}
\end{gathered}
$$

The fragment flying time $T$ can be given by

$$
T=a \frac{\left|\boldsymbol{P} \boldsymbol{M}_{\text {Init_VrelCrd }}\right|}{\left|\boldsymbol{V} \boldsymbol{F}_{\text {Init_TargCrd }}\left(n_{0}\right)\right|} .
$$

The required segments calculation number of each fragment $N_{1}$ can be given by

$$
N_{1}=\operatorname{ceil}\left(\frac{T}{\Delta t_{a t n}}\right) \text {. }
$$

Step 3 Calculate the initial speed and position of each fragment's trajectory segment.

$$
\begin{aligned}
& \boldsymbol{V} \boldsymbol{F}_{\text {TargCrd }}\left(n_{0}, 0\right)=\boldsymbol{V} \boldsymbol{F}_{\text {Init_TargCrd }}\left(n_{0}\right) \\
& \boldsymbol{V} \boldsymbol{F}_{\text {LaunCrd }}\left(n_{0}, 0\right)=\boldsymbol{V} \boldsymbol{F}_{\text {Init_LaunCrd }}\left(n_{0}\right) \\
& \boldsymbol{P F}_{\text {TargCrd }}\left(n_{0}, 0\right)=\boldsymbol{P} \boldsymbol{F}_{\text {Init_TargCrd }}\left(n_{0}\right) .
\end{aligned}
$$

Step 4 This step follows the hit detection algorithm given in [4].

Step 5 Obtain the fragment's each trajectory segment length and the flying time $t_{h t \text { Targ }}$. If it hits the target, $t\left(n_{0}, n_{1}\right)=t_{\text {htTarg }}$; if not, $t\left(n_{0}, n_{1}\right)=\Delta t_{\text {atn }}$, then the flying distance $\boldsymbol{R}\left(n_{0}, n_{1}\right)$ can be given by

$$
\boldsymbol{R}\left(n_{0}, n_{1}\right)=t\left(n_{0}, n_{1}\right)\left|\boldsymbol{V} \boldsymbol{F}_{\text {LaunCrd }}\left(n_{0}, n_{1}-1\right)\right| .
$$

The fragment velocity decay in the launching coordinate system $\boldsymbol{V} \boldsymbol{F}_{\text {LaunCrd }}\left(n_{0}, n_{1}\right)$ can be given by

$$
\begin{gathered}
\boldsymbol{V}_{\text {LaunCrd }}\left(n_{0}, n_{1}\right)=\left[\begin{array}{l}
V F X_{\text {LaunCrd }}\left(n_{0}, n_{1}\right) \\
V F Y_{\text {LaunCrd }}\left(n_{0}, n_{1}\right) \\
V F Z_{\text {LaunCrd }}\left(n_{0}, n_{1}\right)
\end{array}\right]= \\
{\left[\begin{array}{l}
V F X_{\text {LaunCrd }}\left(n_{0}, n_{1}-1\right) e^{-k R\left(n_{0}, n_{1}\right)} \\
V F Y_{\text {LaunCrd }}\left(n_{0}, n_{1}-1\right) e^{-k R\left(n_{0}, n_{1}\right)} \\
V F Z_{\text {LaunCrd }}\left(n_{0}, n_{1}-1\right) e^{-k R\left(n_{0}, n_{1}\right)}
\end{array}\right] \cdot}
\end{gathered}
$$

The fragment velocity decay in the target coordinate system $\boldsymbol{V} \boldsymbol{F}_{\text {TargCrd }}\left(n_{0}, n_{1}\right)$ can be given by

$$
\begin{gathered}
\boldsymbol{V} \boldsymbol{F}_{\text {TargCrd }}\left(n_{0}, n_{1}\right)=\left[\begin{array}{l}
V F X_{\text {TargCrd }}\left(n_{0}, n_{1}\right) \\
V F Y_{\text {TargCrd }}\left(n_{0}, n_{1}\right) \\
V F Z_{\text {TargCrd }}\left(n_{0}, n_{1}\right)
\end{array}\right]= \\
\boldsymbol{E}_{V 2 T} \boldsymbol{E}_{V 2 L}^{-1}\left[\left[\begin{array}{l}
V F X_{\text {LaunCrd }}\left(n_{0}, n_{1}\right) \\
V F Y_{\text {LaunCrd }}\left(n_{0}, n_{1}\right) \\
V F Z_{\text {LaunCrd }}\left(n_{0}, n_{1}\right)
\end{array}\right]-\right.
\end{gathered}
$$




$$
\left.\left[\begin{array}{c}
V X_{T \_L a u n C r d} \\
V Y_{T \_L a u n C r d} \\
V Z_{T \_L a u n C r d}
\end{array}\right]\right] .
$$

\section{Simulation}

\subsection{Sea echo simulation}

The fuze hung on the helicopter flight test can effectively simulate the detection for the fuze flying at very low altitude. We use the test data to verify the sea modeling method mentioned in Section 2.2.3.

The helicopter flies at a speed of $50 \mathrm{~m} / \mathrm{s}$ in the experiment, and the angle between the track and the sea is $20^{\circ}$. The missile's flight attitude, simulation speed and track in the mathematics experimental are completely the same, and the simulation results and the experimental results are shown in Table 1, which can verify that the modeling method is valid.

Table 1 Sea echo test result comparison

\begin{tabular}{cccc}
\hline Item & Altitude/m & Test/dB & Simulation/dB \\
\hline \multirow{2}{*}{ Max energy } & 36.75 & 18 & 17 \\
& 21.10 & 19 & 18 \\
\hline \multirow{2}{*}{ Mean energy } & 36.75 & 8.00 & 6.10 \\
& 21.10 & 7.53 & 7.33 \\
\hline
\end{tabular}

\subsection{Simulation of warhead dynamic power filed}

According to the algorithm described in Section 2.3, the fragment initial velocity is set to $2000 \mathrm{~m} / \mathrm{s}$, and the flying time step is set to $0.3 \mathrm{~ms}$. Missile and target motion parameters at the initiation time are shown in Table 2, conditions without considering fragment trajectory drifting are shown in Fig. 5, the red indicates that the fragment hits the target, and the green represents it does not. The results show that a total of 138 fragments hit the target; the results which consider the fragment trajectory drifting because of the air resistance are shown in Fig. 6. We can see that the fragment trajectory in the relative velocity coordinates is bent. All curves are green, so no fragment hits the target. It can be seen that the trajectory drifting has a significant impact on the assessment results in certain condition.

Table 2 Encounter parameter

\begin{tabular}{cccc}
\hline Item & $X$ & $Y$ & $Z$ \\
\hline Missile velocity/(m/s) & 605.762 & -256.831 & -332.186 \\
Missile position/m & 196385 & 14.779 & -41973.9 \\
Target velocity $/(\mathrm{m} / \mathrm{s})$ & -891.082 & 509.45 & -372.389 \\
Target position $/ \mathrm{m}$ & 196385 & 21.182 & -41982.9 \\
\hline
\end{tabular}

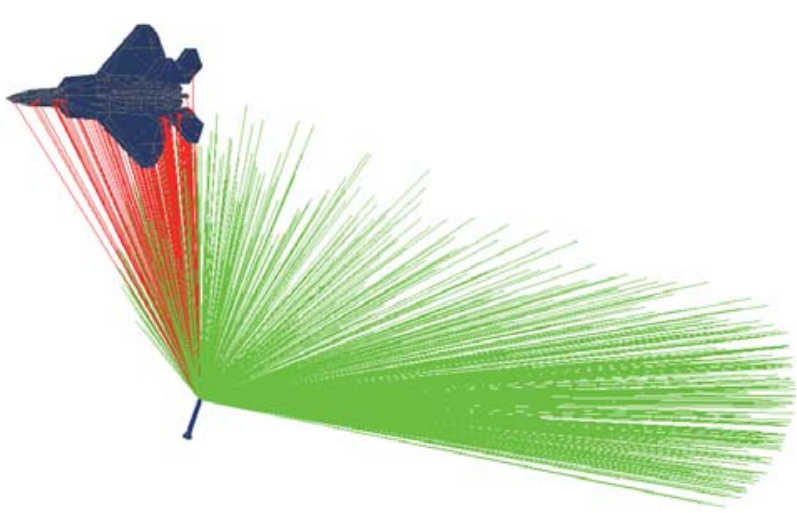

Fig. 5 Hitting target without trajectory drifting

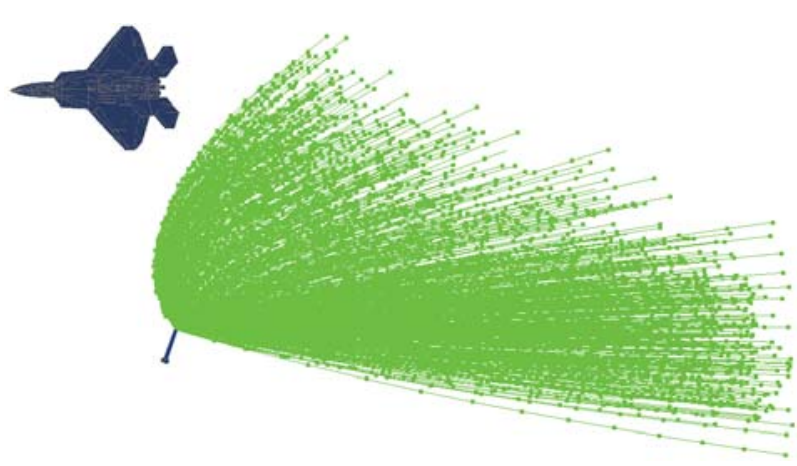

Fig. 6 Hitting target with trajectory drifting

\subsection{Simulation and improvement of FWS}

\subsubsection{Simulation of one particular prototype FWS}

We use the simulation system described previously to simulate and improve a particular FWS. The detection range and warhead power field of the FWS are shown in Fig. 7. The fuze is composed of two detection branches. Each one's function and detection range are not the same. The first antenna branch probe field shown in the green part of Fig. 7 is the lateral bottom of the missile body to detect the target such as encounter position 3 (EP.3) or EP.4, the sea or the ground; the second antenna branch probe field shown in the purple part of Fig. 7 is the lateral top of the missile body to detect the target such as EP.1 or EP.2.

The second antenna branch may detect the sea to cause early burst because the missile body rolls intensely during the flight at very low altitude. The first branch begins to detect the sea when the missile flight altitude decreases. After the sea echo energy exceeds the detection threshold, the fuze adjusts the target detection time domain range according to sea clutter frequency domain range derived from the missile speed. 


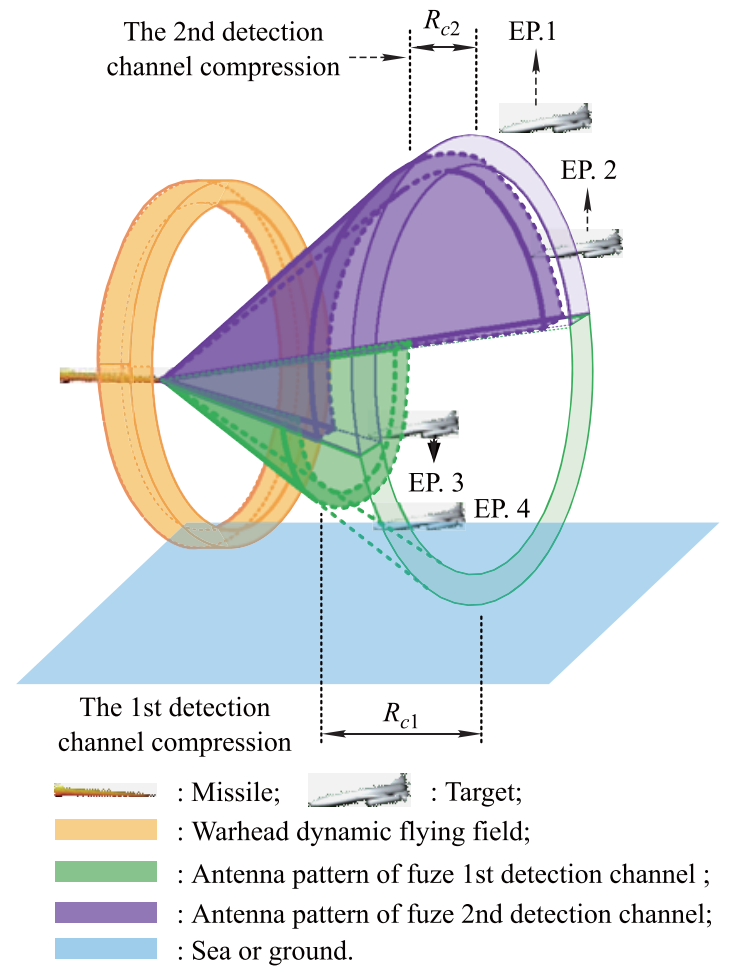

Fig. 7 Particular FWS

The changes of the missile flight altitude and the encounter geometric relation of missile and target are shown in Fig. 8. The fuze is initiated at the moment of the 765 frame, and the missile and target motion parameters at the initiation time are shown in Table 3.

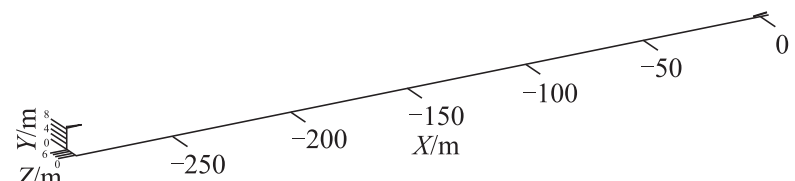

(a) Encounter dispaly in the relative velocity coordinate system

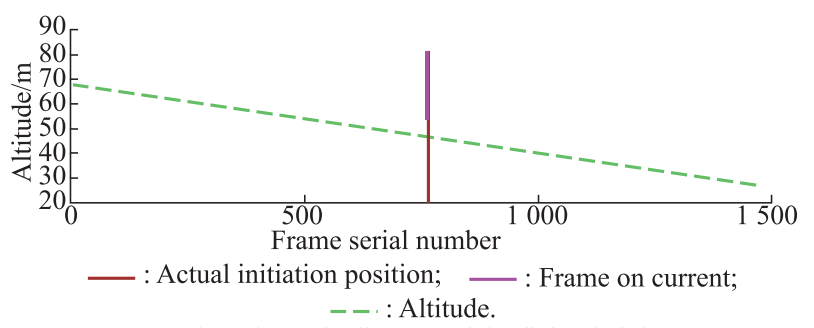

(b) Schematic diagram of the fiying height

Fig. 8 Altitude and encounter geometric relation of missile and target

Table 3 Encounter parameter

\begin{tabular}{cccc}
\hline Item & $X$ & $Y$ & $Z$ \\
\hline Missile velocity $/(\mathrm{m} / \mathrm{s})$ & -813.467 & -90.044 & -708.305 \\
Missile position $/ \mathrm{m}$ & -3978.01 & 47.052 & -3450.39 \\
Target velocity $/(\mathrm{m} / \mathrm{s})$ & 226.413 & 0.261 & 196.817 \\
Target position $/ \mathrm{m}$ & -4189.25 & 20.0002 & -3641.67 \\
\hline
\end{tabular}

The signal detected by the first detection branch is shown in Fig. 9. It does not cause early burst because the first branch can adaptively identify the sea signal and extract the target signal. The signal detected by the second branch is shown in Fig. 10. The signal in this frame causes early burst because it mistakenly detects the sea echo signal as the target signal. No fragment hitting the target is shown in Fig. 11.

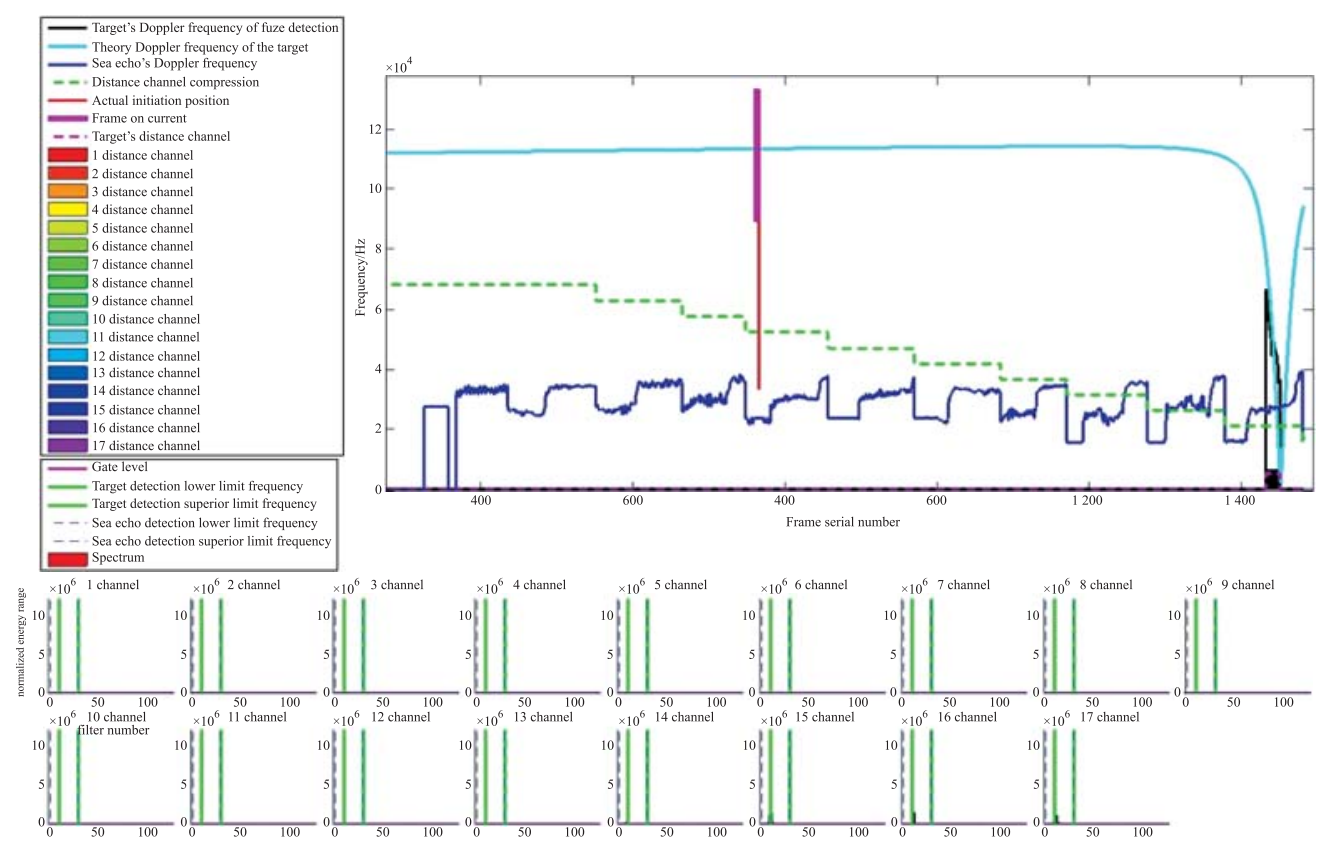

Fig. 9 Signal of the 1st detection branch 


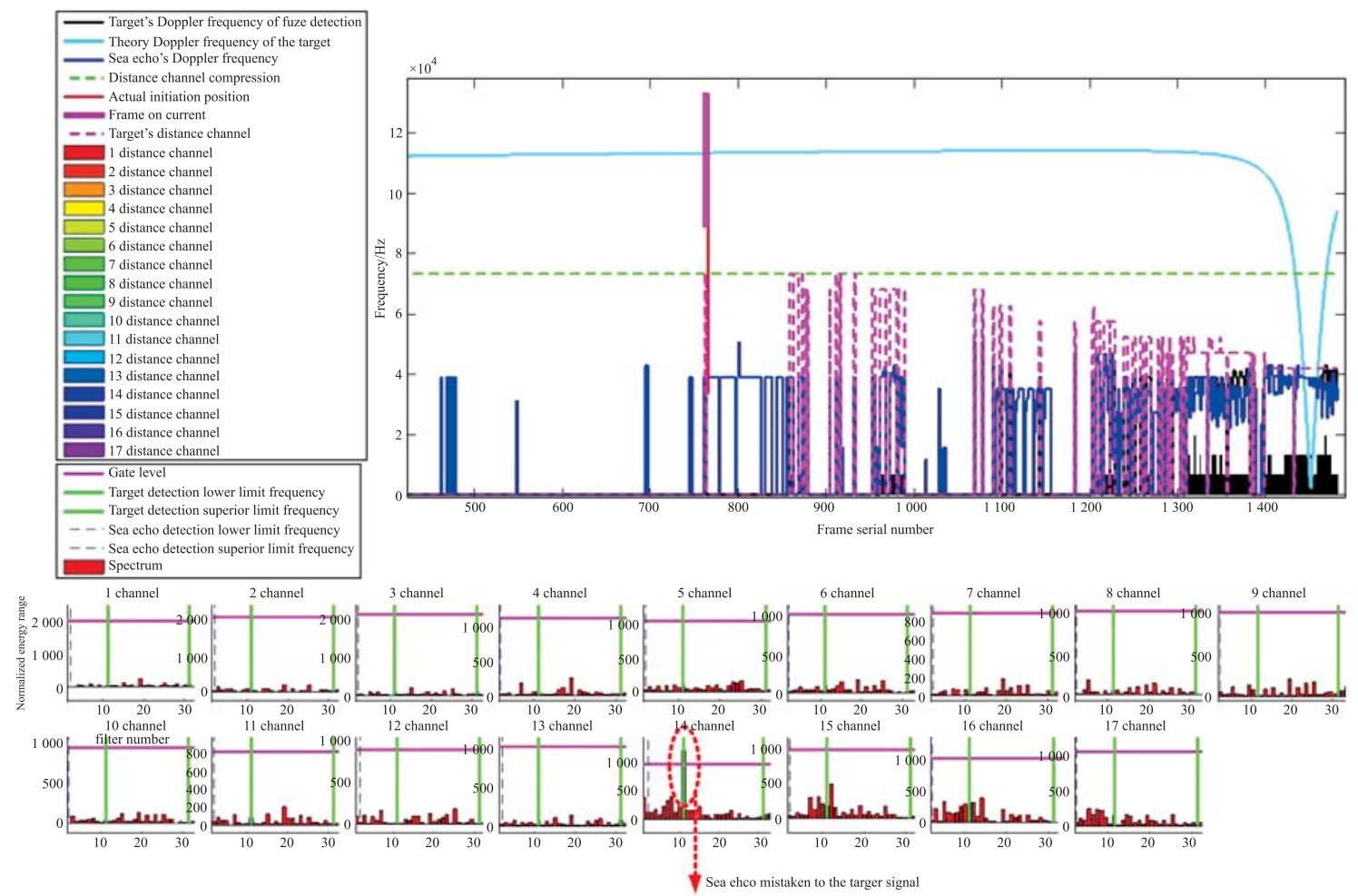

Fig. 10 Signal of the 2nd detection branch

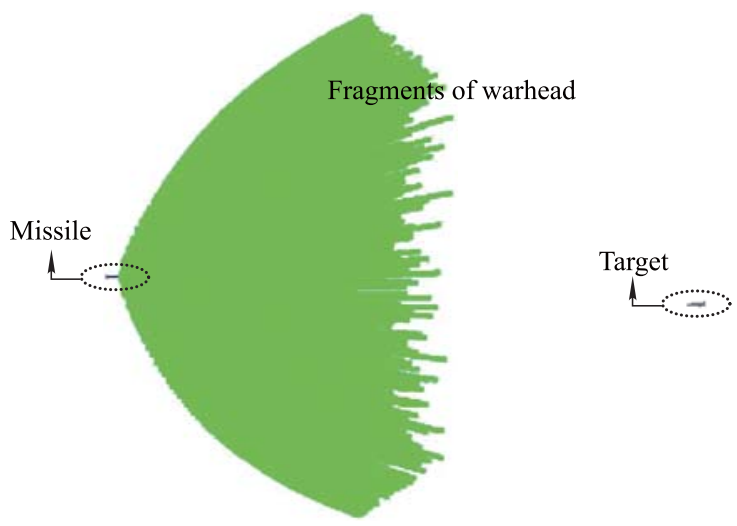

Fig. 11 Early burst of FWS

\subsubsection{Improvement and simulation of FWS}

This section presents a range compression algorithm to solve the problem of fuze mistaken initiation and achieve the maximum of target detection ability.

The algorithm adjusts the second branch target detection in time domain range based on the signal detected by the first detection branch and the missile attitude information.

As shown in Fig. 12, $O X_{g} Y_{g} Z_{g}$ is the ground coordinate system, $O_{m} X_{m} Y_{m} Z_{m}$ is the missile body coordinate system, $X_{m}$ is the missile axes, $\psi_{m}$ is the yaw angle, $\theta_{m}$ is the azimuth angle, $\gamma_{m}$ is the roll angle, $\theta_{1 r}$ and $\theta_{2 r}$ are respectively the angles between the missile axes and the second branch antenna pattern's back and front edges, $R 1$ is the maximum detection distance of second branch, $H$ is the distance between the fuze antenna center and the sea surface, $\alpha_{1 r}$ and $\alpha_{2 r}$ are respectively the angles between the sea surface and the second branch antenna pattern's back and front edges.

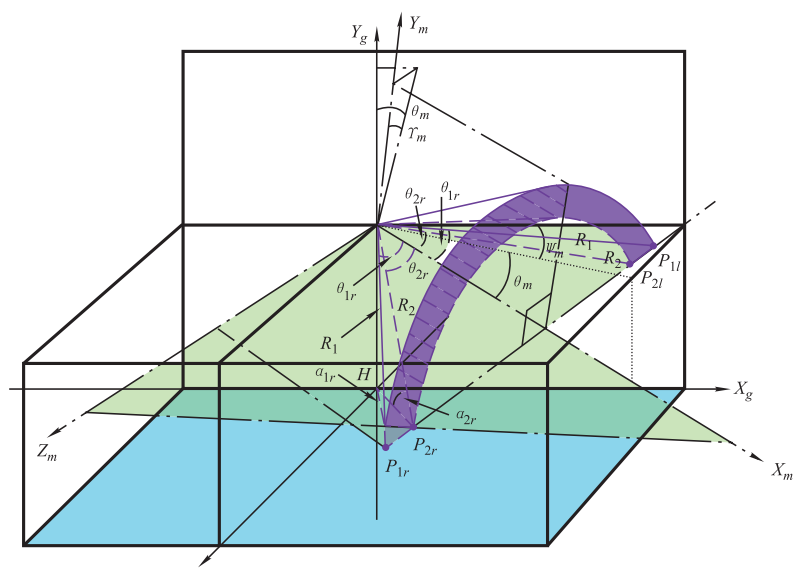

Fig. 12 Improved algorithm of the 2nd branch

In Fig. $12, \alpha_{1 r}$ can be given by

$$
\alpha_{1 r}=\arcsin \left(H / R_{1}\right) .
$$

The coordinates of the edge on both sides of the beam pattern in the missile body coordinate system can be given 
by

$$
\begin{aligned}
& {\left[\begin{array}{c}
X_{1 m_{-} r} \\
Y_{1 m_{-} r} \\
Z_{1 m_{-} r}
\end{array}\right]=\left[\begin{array}{c}
R_{1} \cos \theta_{1 r} \\
0 \\
R_{1} \sin \theta_{1 r}
\end{array}\right]} \\
& {\left[\begin{array}{l}
X_{1 m_{-} l} \\
Y_{1 m_{-} l} \\
Z_{1 m_{-} l}
\end{array}\right]=\left[\begin{array}{c}
R_{1} \cos \theta_{1 r} \\
0 \\
-R_{1} \sin \theta_{1 r}
\end{array}\right]} \\
& {\left[\begin{array}{l}
X_{2 m \_} r \\
Y_{2 m \_r} \\
Z_{2 m \_r}
\end{array}\right]=\left[\begin{array}{c}
R_{2} \cos \theta_{2 r} \\
0 \\
R_{2} \sin \theta_{2 r}
\end{array}\right]} \\
& {\left[\begin{array}{c}
X_{2 m \_} \\
Y_{2 m \_l} \\
Z_{2 m \_l}
\end{array}\right]=\left[\begin{array}{c}
R_{2} \cos \theta_{2 r} \\
0 \\
-R_{2} \sin \theta_{2 r}
\end{array}\right] .}
\end{aligned}
$$

The transform matrix from the missile body to the ground coordinate system can be given by

$$
\boldsymbol{M}_{M 2 G}=\boldsymbol{M}_{y}\left(-\psi_{m}\right) \cdot \boldsymbol{M}_{z}\left(-\theta_{m}\right) \cdot \boldsymbol{M}_{x}\left(-\gamma_{m}\right) .
$$

We can get the coordinates of the edge on both sides of the beam pattern in the ground coordinate system as

$$
\begin{aligned}
& {\left[\begin{array}{c}
X_{1 g_{-} r} \\
Y_{1 g_{\_} r} \\
Z_{1 g_{\_} r}
\end{array}\right]=M_{M 2 G}\left[\begin{array}{c}
X_{1 m_{-} r} \\
Y_{1 m_{-} r} \\
Z_{1 m_{-} r}
\end{array}\right]} \\
& {\left[\begin{array}{c}
X_{1 g_{-} l} \\
Y_{1 g_{-} l} \\
Z_{1 g_{-} l}
\end{array}\right]=M_{M 2 G}\left[\begin{array}{c}
X_{1 m_{-} l} \\
Y_{1 m_{-} l} \\
Z_{1 m_{-} l}
\end{array}\right] .}
\end{aligned}
$$

The angle in the ground coordinate system between the edge of the beam pattern and the sea surface $\alpha$ according to the geometry relation can be given by

$$
\alpha=\max \left\{\alpha_{1 r}, \alpha_{1 l}, \alpha_{2 r}, \alpha_{2 l}\right\}
$$

where

$$
\alpha_{1 r}=-\arcsin \left(\frac{Y_{1 g \_r}}{R_{1}}\right)=
$$

$$
\begin{gathered}
\arcsin \left[\sin \left(-\theta_{m}\right) \cos \theta_{1 r}-\cos \left(-\theta_{m}\right) \sin \left(-\gamma_{m}\right) \sin \theta_{1 r}\right] \\
\alpha_{1 l}=-\arcsin \left(\frac{Y_{1 g_{-} l}}{R_{1}}\right)=
\end{gathered}
$$

$\arcsin \left[\sin \left(-\theta_{m}\right) \cos \theta_{1 r}+\cos \left(-\theta_{m}\right) \sin \left(-\gamma_{m}\right) \sin \theta_{1 r}\right]$

$$
\alpha_{2 r}=-\arcsin \left(\frac{Y_{2 g \_r}}{R_{2}}\right)=
$$

$\arcsin \left[\sin \left(-\theta_{m}\right) \cos \theta_{2 r}-\cos \left(-\theta_{m}\right) \sin \left(-\gamma_{m}\right) \sin \theta_{2 r}\right]$

$$
\alpha_{2 l}=-\arcsin \left(\frac{Y_{2 g_{-} l}}{R_{2}}\right)=
$$

$\arcsin \left[\sin \left(-\theta_{m}\right) \cos \theta_{2 r}+\cos \left(-\theta_{m}\right) \sin \left(-\gamma_{m}\right) \sin \theta_{2 r}\right]$.

$$
N_{2}=\text { floor }\left(\frac{d_{1} N_{1}-d_{\text {safe }}}{d_{2} \sin \alpha}\right)
$$

where $d_{\text {safe }}$ is the safe distance, $d_{1}$ and $d_{2}$ are respectively the first and second branch distance channel range, $N_{1}$ and $N_{2}$ are respectively sizes of the first and second branch distance channel.

Fig. 13 is the simulation result based on the improved FWS, where the green line represents the time domain detection range compression, and we can see the difference comparing with Fig. 10. The improved FWS does not cause early burst as shown in Fig. 13 and Fig. 14 and a total of 107 fragments hit the target. The missile and target motion parameters at the initiation time are shown in Table 4.

Table 4 Encounter parameter

\begin{tabular}{cccc}
\hline Item & $X$ & $Y$ & $Z$ \\
\hline Missile velocity $/(\mathrm{m} / \mathrm{s})$ & -819.013 & -100.806 & -735.321 \\
Missile position $/ \mathrm{m}$ & -4143.03 & 28.3348 & -3596.03 \\
Target velocity $/(\mathrm{m} / \mathrm{s})$ & 226.413 & 0.258 & 196.817 \\
Target position $/ \mathrm{m}$ & -4143.54 & 19.9961 & -3601.94 \\
\hline
\end{tabular}
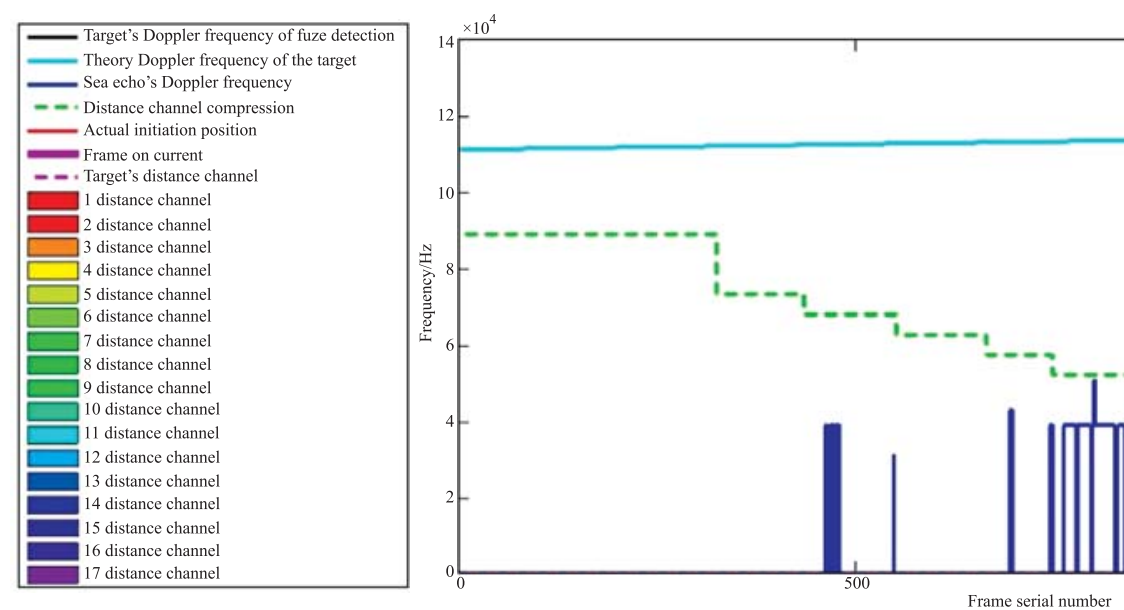

Fig. 13 Signal results of the improved FWS 

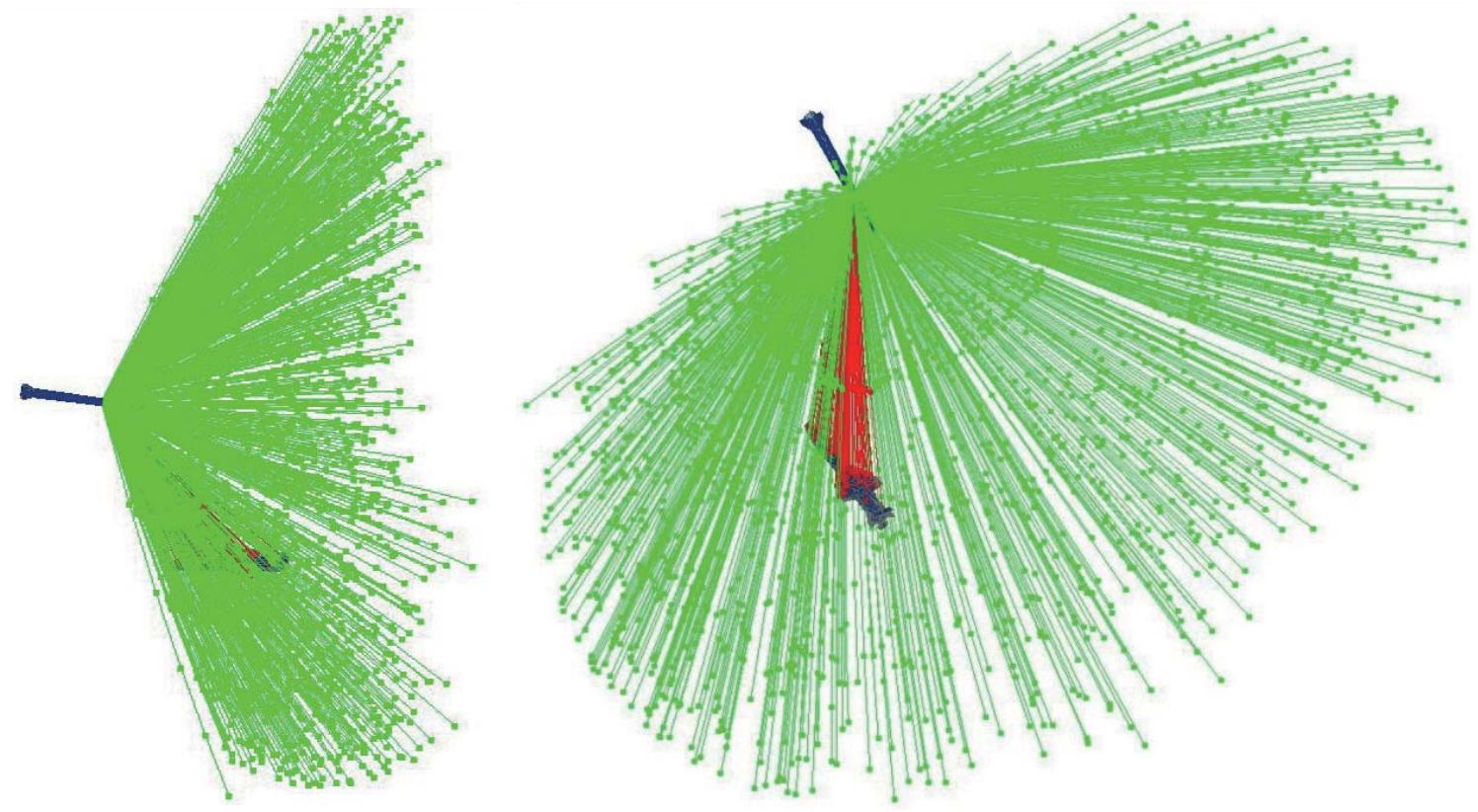

Fig. 14 Killing target by the improved FWS

\section{Conclusions}

In this paper, a novel FWS including a novel general fuze model suitable for the complicated antenna pattern, a novel sea echo model which can combine with the target multiscattering centers model to simulate the mixed echoes and a warhead dynamic effectiveness power field model which can simulate the fragment trajectory drifting is proposed. Compared with the traditional methods, the proposed simulation system is more accurate for the FWS flying at very low altitude. Sea echo simulation experiment can verify the sea echo modeling method is valid. A typical experiment shows that the trajectory drifting has a significant impact on the assessment results in certain condition. Finally, a particular FWS is simulated using this simulation system, early burst is found and the corresponding improvement is made according to the results. The improved FWS can hit the target effectively, which proves the proposed system is valid and practical.

\section{References}

[1] Y. L. Liu, Y. Zhang. Summarization of low altitude fuze technology. Guidance \& Fuze, 2010, 31(4): 1 -4. (in Chinese)

[2] C. H. Zhou, J. G. Li. The simulation and modeling of sea clutter received by the radio pulse fuzes. Guidance \& Fuze, 2010, 31(3): 18-22. (in Chinese)

[3] J. Feng, J. Cao. Missile radio fuze echo simulation based on bright spot model. Modern Defense Technology, 2008, 36(5): 32-36. (in Chinese)

[4] B. Niu, L. X. Gu. Design and application of a kind fuzewarhead coordination simulation system based on modularization idea. Journal of System Simulation, 2009, 21(23): 7452 7455. (in Chinese)
[5] W. X. Xu, Z. D. Song. Research on terminal damage model and simulation of multi-purose guided missile warhead attacking on helicopter. Acta Armamentari, 2007, 28(6): 671-676. (in Chinese)

[6] B. Niu, L. X. Gu, C. L. Gong. Assessment of lethality of fragment warhead to armed-helicopter. Journal of Ballistics, 2011, 23(1): 68 - 71. (in Chinese)

[7] Y. X. Wang, Y. H. Cheng, Z. Yang. A method to improve fuze warhead matching efficiency of missile intercepting high velocity and minimum altitude target. Modern Defense Technology, 2009, 37(5): 14-17. (in Chinese)

[8] J. F. Dammam. Air target models for fuzing simulation. Harry Diamond Laboratories, US Army Electronics Research and development. ADA121473. 1982-9.

[9] S. W. Lee, J. AndershD, R. Kipp, et al. Near-field radar signature modeling for ew/end-game simulations. ADA318778. Naval Air Warfare Center Weapons DIV Point MUGU CA, 1996.

[10] L. N. Medgyesi-Mitschang, P. G. Moore, D. L. SMITH, et al. Ultra-near field facility for RCS research. Washington D. C. : Naval Research Laboratory, 2001.

[11] J. Cao Application of highlight method to target echo computation in fuse simulation. Modern Defense Technology, 2007, 35(3): 46-48. (in Chinese)

[12] Q. Zhao. Research On the characteristics of near field aerial target based on multi-scattering centers. Beijing, China: Beijing Institute of Technology, 2009. (in Chinese)

\section{Biographies}

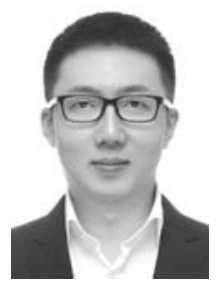

Qi Zhao was born in 1981. Currently he is a senior engineer in Beijing Institute of Electronic System Engineering. He received his B.S. degree from School of Information and Communication, Harbin Engineering University in 2004, majored in communication engineering. He took a successive postgraduate and doctoral program of study from 2004 and received his Ph.D. degree in 2009 from 
School of Information and Electronics, Beijing Institute of Technology (BIT), majored in electromagnetic field and microwave technology. His main research interests include system design, simulation and analysis. E-mail: zhaoqineo@hotmail.com

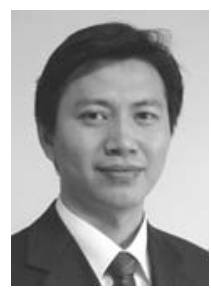

Guangyu Du was born in 1973. Currently he is a researcher in Beijing Institute of Electronic System Engineering. He received his B.S. degree from Department of Electronics information Engineering, Beihang University in 1997, majored in radio guidance. He received his M.S. degree from University of Chinese Academy of Sciences in 2005, majored in communication and information system. He is currently a Ph.D. candidate in Beijing Institute of Electronic System Engineering, majored in guidance and control. His main research interests include system design and simulation.

E-mail: another2009@ sina.com

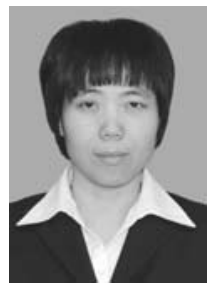

Bin Zhang was born in 1968. Currently she is a researcher in Beijing Institute of Electronic System Engineering. She received her B.S. degree from School of Electronics Engineering, Tianjin University in 1990, and her M.S. degree from School of Mechanical and Electronic Engineering, Beijing Institute of Technology in 1993, majored in fuze technology. Her main research interests include fuze and warhead system design and simulation of air defense missile.

E-mail: zxhzb9092@126.com

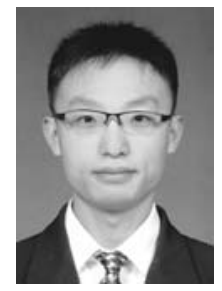

Yanbin Zhai was born in 1989. Currently he is an associate engineer in Beijing Institute of Electronic System Engineering. He received his B.S. degree in 2011, and his M.S. degree in 2013 from School of Astronautics, Harbin Institute of Technology, majored in control science and engineering. His main research interests are the design of fuze warhead matching, and simulation design of fuze warhead system of air defense missile.

E-mail: zhaiyanbin0828@gmail.com

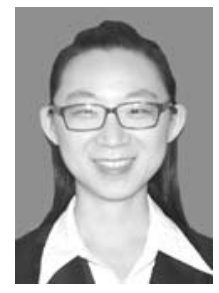

Yuruo Shi was born in 1989. Currently she is an associate engineer in Beijing Institute of Electronic System Engineering. She received her B.S. degree from the Institute of Mathematic and Statistics, Wuhan University in 2011, majored in information and computational science. She received her M.S. degree from Beijing Institute of Electronic System Engineering in 2014, majored in flight vehicle design. Her research interests are the design of fuze warhead matching, simulation design of fuze warhead system of air defense missile.

E-mail: syr13.student@sina.com 